Proc. of the X Int. Conf. - Ion Implantation and other Applications of Ions and Electrons, Kazimierz Dolny 2014

\title{
Calculations of Ion Beam Emittance for Hot Cavity Ion Source
}

\author{
M. TUREK* \\ Institute of Physics, Maria Curie Skłodowska University, pl. M. Curie-Skłodowskiej 1, 20-031 Lublin, Poland \\ Emittance of short-lived nuclide beams produced in hot cavity ion sources is calculated. Influence of half- \\ life period as well as the average sticking time on beam emittance is under investigation. Two different shapes of \\ ionizer cavity are considered: almost fully spherical and hemispherical ones. Changes of beam emittance due to the \\ extraction channel geometry (its diameter and length) are studied. A new concept of scaled efficiency (ion source \\ brightness analogon) is introduced in order to compare the two-ion source configurations. Phase space portraits of \\ the extracted beams are presented.
}

DOI: 10.12693/APhysPolA.128.931

PACS: 07.77.Ka, 07.05.Tp, 34.35.+a, 41.75.Ak, 41.75.Cn

\section{Introduction}

Obtaining high-intensity particle beams is crucial in many fields of experimental physics (high-energy and accelerator physics as well as nuclear spectroscopy etc.). Not only the intensity of the ion beam is important but also its quality measured e.g. by its divergence/convergence and energy spread. Numerical simulations are widely used in order to design high-current ion sources characterized by possibly low transverse emittance [1-4].

In the paper there is considered a hot cavity ion source, a device that has construction designed in 1970s [5] but constantly developed $[6-8]$ due to its numerous merits like beam purity, high efficiency and robustness. A lot of analytical models were developed over decades ([9-11], see also references in [12]). Some attempts were made in order to describe diffusion of nuclides in target and effusion in the surface ion sources [13-15]. Recently, a numerical model of ionization in hot cavity ion source has been developed $[12,16,17]$, taking into account not only surface ionization but also possible effects of electron impact ionization mechanism [18]. Initially the model was suitable for stable and long-lived isotopes, but radioactive decay and delays due to the diffusion and effusion were implemented [12, 19], making it useful also for short-lived isotopes, which are common in on-line nuclear spectroscopy. Mainly tubular cavities were considered, but the model was extended to spherical cavities [20], which are employed in experiments [21, 22].

The paper contains brief description of both the numerical model used for simulation and rms emittance formulae, given for completeness. Dependences of beam emittance on nuclide surface ionization probability, halflife period and its average sticking time are presented and discussed. Two different shapes of the ionizer cavity are considered as in [20]. Influence of the extraction

*e-mail: mturek@kft.umcs.lublin.pl opening geometry (i.e. the diameter and the length of the extraction channel) is considered. A new concept of ion source scaled efficiency (a magnitude similar to brightness) is introduced in order to compare merits of the two above mentioned ionizer cavity configurations. Phase space beam portraits are presented for both ionizer configurations.

\section{Beam emittance}

A beam consisting of $N$ particles could be described by a point in $6 N$-dimensional canonical phase space $\left(x, y, z, p_{x}, p_{y}, p_{z}\right)$. Alternatively, a group of $N$ points in the 6-dimensional phase space could be considered. In the case of a bunch of particles moving in approximately one direction (say $z$ ), it is convenient to decouple longitudinal and transversal motion. Finally, 4D transversal phase space could be divided into two 2 D subspaces (say vertical and horizontal ones). The momentum along the beam axis $\left(p_{z}\right)$ is much greater than the transversal component. Hence, it is convenient to consider orbital angles rather than momenta

$$
x^{\prime}=\frac{p_{x}}{p_{z}} \text { and } y^{\prime}=\frac{p_{y}}{p_{z}} .
$$

Transverse fractional emittances are defined as the surface of the ellipses containing some rather arbitrary chosen part of the beam (usually $99 \%, 95 \%$ or $90 \%$ ) in the $x x$ ' and $y y$ 'subspaces (usually divided by $\pi$ ). The emittance ellipse axes are parallel to $x$ and $x^{\prime}$ ones, the ellipse main semi-axes could be understood as the beam half-size $\Delta x$ and beam divergence $\Delta x^{\prime}$.

The above definition is stylish, but that of rms emittance given in $[23,24]$ is more useful for numerical simulation purposes

$$
\begin{aligned}
& \varepsilon_{x, \mathrm{rms}}=\sqrt{\left\langle x^{2}\right\rangle\left\langle x^{\prime 2}\right\rangle-\left\langle x x^{\prime}\right\rangle^{2}} \\
& \quad \text { and } \varepsilon_{y, \mathrm{rms}}=\sqrt{\left\langle y^{2}\right\rangle\left\langle y^{\prime 2}\right\rangle-\left\langle y y^{\prime}\right\rangle^{2}},
\end{aligned}
$$

where brackets mean the average on the ensemble. In order to take into account particles outside the rms ellipse, some authors suggest multiplying rms emittance by the factor of 4 . 


\section{Numerical model}

The numerical model of ionization in hot cavities was previously described in detail. The basic facts are reminded here for completeness. The code track test particles integrate numerically the classical equations of motion. The electrostatic potential determined by electrodes potentials and shapes is found by solving the Laplace equation with the successive over-relaxation technique, as in [25-29]. The flat extraction electrode is placed at the distance $d$ from the extraction opening. The geometry of the ionizer is shown in Fig. 1.

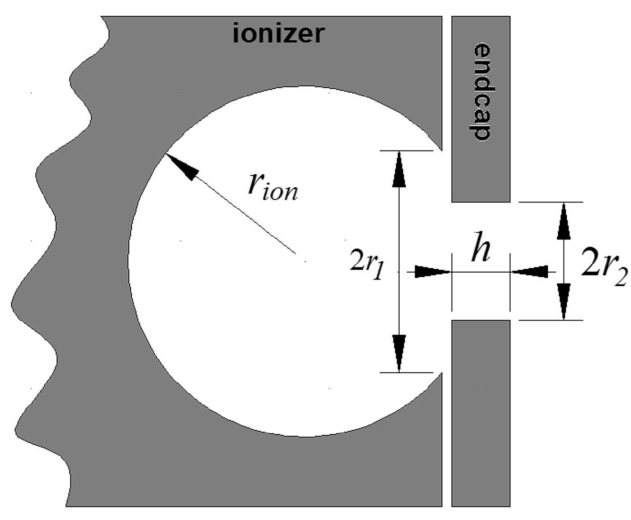

Fig. 1. Geometry of the ionizer cavity.

A particle could be ionized/neutralized at the hot ionizer surface with probability given by the ionization coefficient $\beta$, see [12] for details. Nuclides produced in the ion source undergo radioactive decay, hence each test particle is assumed to transform from primary to secondary nuclide after some time $t_{\mathrm{dec}}$ determined using the Monte Carlo approach

$$
t_{\mathrm{dec}}=-\tau_{1 / 2} \ln (\mathrm{RND}),
$$

where $\tau_{1 / 2}$ is the primary nuclide half-life period and RND is the normal pseudorandom number. The time a particle stays in the cavity depends not only on a flight time but also on the time a particle spends on the hot surface during each collision

$$
t_{\text {stick }}=-\tau_{\mathrm{s}} \ln (\mathrm{RND}),
$$

where $\tau_{\mathrm{s}}$ is the particle average sticking time. The initial velocity direction of the particle leaving the surface is random and its value corresponds to the ionizer temperature.

Particles are tracked until they reach the outer rim of the extraction channel. The extracted beam emittance is then calculated according to the formula (2).

\section{Results}

Calculations were done for two different shapes of cavity. The first one (configuration A) is a spherical cavity with rather small extraction opening. Both $r_{1}$ and $r_{2}$ radii are the same $\left(r_{1}=r_{2}=0.5 \mathrm{~mm}\right.$, unless otherwise specified) and four times smaller than the radius of the cavity $r_{\text {ion }}=2 \mathrm{~mm}$. The hemispherical configuration (B) could be defined by the following choice: $r_{1}=r_{\text {ion }}$ and $r_{2}<r_{1}$. As previously, $r_{\text {ion }}=2 \mathrm{~mm}$ and $r_{2}=0.5 \mathrm{~mm}$ were chosen, unless otherwise specified. The extraction electrode on the potential $V_{\text {ext }}=-2 \mathrm{kV}$ was placed at the distance $d=2 \mathrm{~mm}$ from the outer rim of the extraction channel. The ionizer temperature was set to $k T=0.31 \mathrm{eV}$. Figure $2 \mathrm{a}$ and b shows changes of the extracted beam emittance with the ionization coefficient for different values of the half-life. Simulations were done using 200000 of test particles of mass 150 a.m.u. The simulation time-step was $10^{-8} \mathrm{~s}$. The length of the extraction channel was $h=0.1 \mathrm{~mm}$.

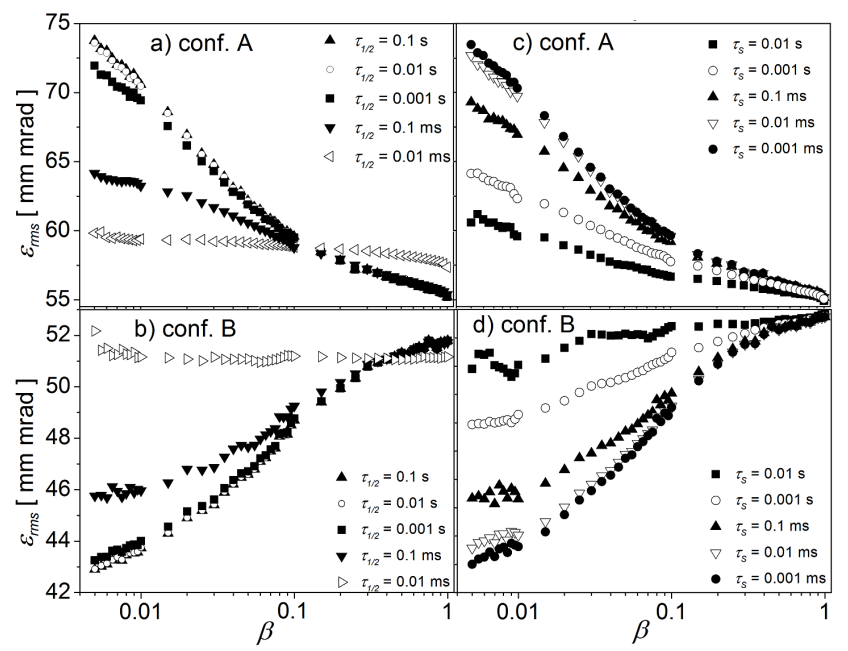

Fig. 2. $\quad \varepsilon_{\mathrm{rms}}(\beta)$ curves for different nuclide half-life periods (sticking neglected) and analogous curves for different $\tau_{\mathrm{s}}$ values $\left(\tau_{1 / 2}=0.01 \mathrm{~s}\right)$ for almost fully spherical (a) and (c) and hemispherical ionizers (b) and (d).

First of all, the configuration B yields a beam of smaller emittance, below $52 \mathrm{~mm}$ mrad for all parameter choices considered. This could possibly be due to the focusing effect of the hemispherical ionizing surface. On the other hand, beam emittances for the configuration $\mathrm{A}$ are in the range $55-75 \mathrm{~mm} \mathrm{mrad}$. One can see completely different behaviour of $\varepsilon_{\mathrm{rms}}(\beta)$ curves for the two configurations. In the case $A$ there is observed a decrease of emittance with $b$, while for the configuration $\mathrm{B}$ the trend is opposite. The increase of emittance for large $\beta$ in the case $\mathrm{B}$ could be explained by the contribution from the ions produced during the first contact with the surface in the outer region of the hemisphere. They have a much larger transversal momentum than the particles emitted from the surface near the axis of the cavity. For small $\beta$ the probability of ionization during the first collision decreases and the particles with a large transversal momentum pass the extraction opening as neutral atoms and do not count as ion beam emittance.

The nuclide half-life changed in the range from $0.01 \mathrm{~ms}$ up to $0.1 \mathrm{~s}$. In the case $A$ one observes lowering of $\varepsilon_{\mathrm{rms}}(\beta)$ with the decreasing $\tau_{1 / 2}$ (improving beam quality) while 
the reverse trend could be seen for the configuration $B$. In both cases the changes of emittance with $\tau_{1 / 2}$ were most pronounced for small $\beta(\beta<0.1)$. Note that the slopes of $\varepsilon_{\text {rms }}(\beta)$ curves are steeper for longer $\tau_{1 / 2}$ and the differences of performances for both configurations are approximately $20 \%$ for very short-lived nuclides.

Influence of average sticking time on the beam emittance was also investigated. Calculations were done for $\tau_{1 / 2}=0.01 \mathrm{~s}$ and $\tau_{\mathrm{s}}$ changing in the range from $1 \mu \mathrm{s}$ up to $0.01 \mathrm{~s}$. All other simulation parameters were kept the same as in the previous case. One can see in Fig. 2c and $\mathrm{d}$ that in the case $\mathrm{A}$ emittance decreases with rising $\tau_{\mathrm{s}}$ while in the case B emittance rises when particles stay longer in the ionizer. The slopes of $\varepsilon_{\text {rms }}(\beta)$ curves are smallest for the case when particles stay long inside the cavity $\left(\tau_{\mathrm{s}}=10 \mathrm{~ms}\right)$. Emittance for the hemispherical configuration is again $\approx 20 \%$ smaller than that for the spherical one.

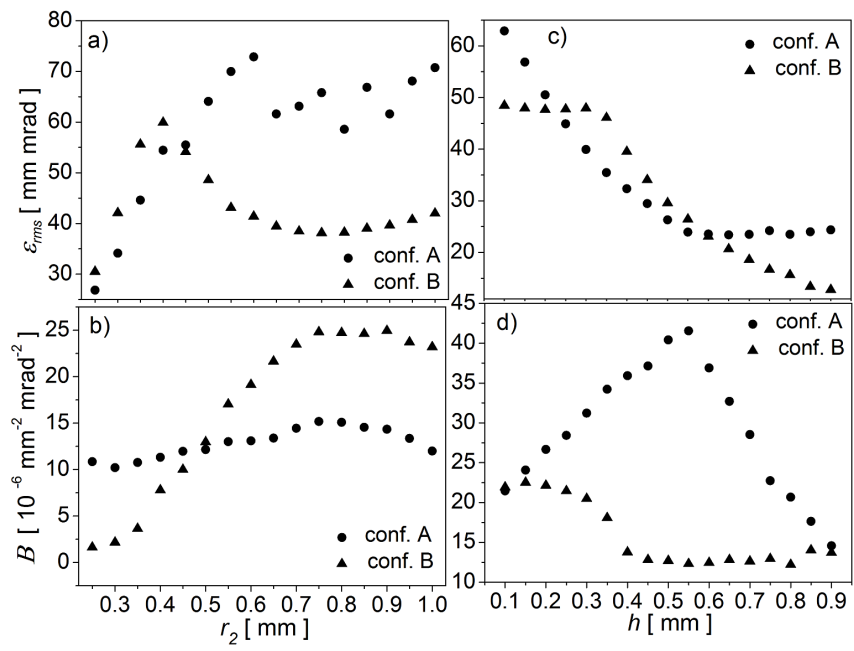

Fig. 3. Dependences of rms emittance (a) and (c) and scaled efficiency (b) and (d) on the extraction opening radius (upper part) and the length of extraction channel (lower part).

Changes of beam emittance with the radius of extraction hole are shown in Fig. 3a. Average sticking time was set to $1 \mathrm{~ms}$. In the case of spherical ionizer one can see the increase of emittance followed by a saturation (at a level of $\approx 65 \mathrm{~mm} \mathrm{mrad}$ ) for $r_{2}$ larger than $0.6 \mathrm{~mm}$. The behaviour of $\varepsilon_{\mathrm{rms}}\left(r_{2}\right)$ curve is more complex - one can see a maximum at $r_{2}=0.4 \mathrm{~mm}$, then emittance falls below $45 \mathrm{~mm}$ mrad. The better performance of configuration $\mathrm{B}$ for larger extraction opening could be due to the fact that the area penetrated by the extraction voltage is larger (than in the case A) compared to the whole volume of the ionizer cavity. Similar results were obtained for other $\tau_{1 / 2}$.

In analogy to brightness, which is one of the basic figures of merits for the ion source one can introduce scaled efficiency

$$
B=\frac{\beta_{\mathrm{s}}}{\varepsilon_{x, \mathrm{rms}} \varepsilon_{y, \mathrm{rms}}},
$$

relating the efficiency of the ion source to the phase space volume of the produced beam. Figure $3 \mathrm{~b}$ shows the changes of $\mathrm{B}$ with the extraction opening radius. The scaled efficiency of the configuration $\mathrm{B}$ is higher than that of configuration A, mostly due to very low emittance for larger $r_{2}$. On the other hand, one should notice that spherical ionizer prevails for small $r_{2}$, mostly due to much higher $\beta_{\mathrm{s}}$.

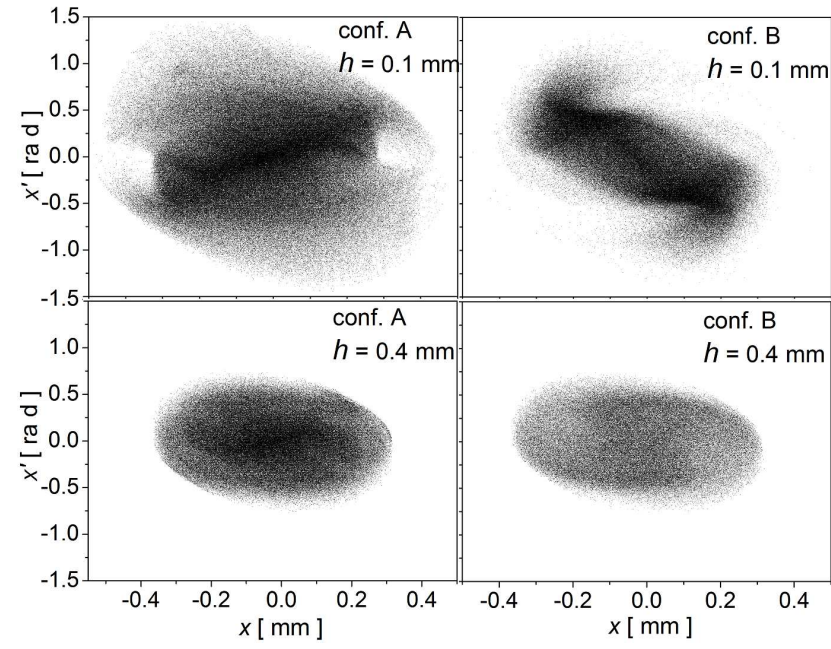

Fig. 4. Examples of phase space beam portraits for both ionizer configurations.

One may expect that the length of extraction channel may influence both the emittance (the larger $h$, the smaller are the transversal momenta of extracted particles) and the efficiency of ionization (long extraction channel means worse penetration of the extraction field). The trade-off between the two competing trends should be found. Figure $3 \mathrm{c}$ shows the $\varepsilon_{\text {rms }}(h)$ curve calculated for $r_{2}=0.5 \mathrm{~mm}$ and $h$ up to $0.9 \mathrm{~mm}$. In both cases emittance decreases with the length of the channel. However, there is a saturation in the case of configuration $\mathrm{A}$ for $h>0.5 \mathrm{~mm}$ (at the level of $25 \mathrm{~mm} \mathrm{mrad}$ ), while for the hemispherical ionizer emittance still decreases reaching values below $15 \mathrm{~mm}$ mrad. On the other hand, there is a plateau for $h<0.3 \mathrm{~mm}$ (case B). Differences of $\varepsilon_{\text {rms }}(h)$ curve shapes combined with the fact that efficiency of configuration $\mathrm{A}$ is higher, result in quite different $B(h)$ shapes, shown in Fig. 3d. The scaled efficiency of the spherical configuration (A) is much higher, up to 3 times in the maximum (for $h=0.55 \mathrm{~mm}$ ). The larger values of $B$ are achieved for smaller $h(h<0.3 \mathrm{~mm})$. Figure 4 shows phase space portraits of beams passing the extraction opening for the two shape configurations. The tilt of inner, darker part obtained for the configuration $\mathrm{A}$ and $h=0.1 \mathrm{~m}$ suggests beam divergence, while for the hemispherical ionizer the beam is rather convergent. For larger $h$ both beams become more parallel, as the emittance ellipse is placed along the $x$ axis. 


\section{Conclusions}

Emittance calculations for particle beams produced in the spherical hot cavity ion source were presented. Two different ionizer shape configurations were considered: one almost fully spherical and hemispherical. It was found that in the first case beam emittance increases with the surface ionization probability, while in the second one the trend is opposite. Emittance decreases for a short half-life period when spherical ionizer is considered, while it increases for the hemispherical cavity. Different behavior with the average sticking time is also observed. Generally, the beam emittance is better by several tens of percent for hemispherical ionizer. However, the newly introduced figure of merit, named scaled efficiency shows that spherical ionizer is superior in a wide range of parameters, e.g. for extraction opening radius smaller than $0.5 \mathrm{~mm}$.

\section{References}

[1] P. Chen, R. Yi, D. Yu, in: IEEE Particle Accelerator Conf., Albuquerque 2007, p. 3684.

[2] M.M. Abdelrahman, S.G. Zakhary, Braz. J. Phys. 39, 275 (2009).

[3] D. Winklehner, D. Todd, J. Benitez, M. Strohmeier, D. Grote, D. Leitner, J. Instrument. 5, P12001 (2010).

[4] H.-J. Kwon, H.-S. Kim, D.-I. Kim, J.-H. Jang, Y.S. Cho, J. Kor. Phys. Soc. 56, 1998 (2010).

[5] G.J. Beyer, E. Herrmann, A. Piotrowski, V.I. Raiko, H. Tyroff, Nucl. Instrum. Methods 96, 437 (1971).

[6] V.N. Panteleev, Rev. Sci. Instrum. 75, 1602 (2004).

[7] T. Stora, Nucl. Instrum. Methods Phys. Res. B 37, 402 (2013).

[8] M. Manzolaro, A. Andrighetto, G. Meneghetti, M. Rossignoli, S. Corradetti, L. Biasetto, D. Scarpa, A. Monetti, S. Carturan, G. Maggioni, Nucl. Instrum. Methods Phys. Res. B 317, 446 (2013).

[9] A. Latuszyński, V.I. Raiko, Nucl. Instrum. Methods 125, 61 (1975).

[10] V.P. Afanas'ev, V.A. Obukhov, V.I. Raiko, Nucl. Instrum. Methods 145, 533 (1977).
[11] A. Latuszyński, K. Pyszniak, A. Droździel, M. Turek, D. Mączka, J. Meldizon, Vacuum 81, 1150 (2007).

[12] M. Turek, Vacuum 104, 1 (2014).

[13] Y. Zhang, G.D. Alton, J. Vac. Sci. Technol. A 23, 1558 (2005).

[14] B. Mustapha, J.A. Nolen, Nucl. Instrum. Methods Phys. Res. A 521, 59 (2004).

[15] Y. Zhang, I. Remec, G.D. Alton, Z. Liu, Nucl. Instrum. Methods Phys. Res. A 620, 142 (2010).

[16] M. Turek, K. Pyszniak, A. Drozdziel, J. Sielanko, Vacuum 82, 1103 (2008).

[17] M. Turek, A. Drozdziel, K. Pyszniak, D. Maczka, B. Slowinski Rev. Sci. Instrum. 83, 023303 (2012).

[18] M. Turek, K. Pyszniak, A. Droździel, Vacuum 83, S260 (2009).

[19] M. Turek, Acta Phys. Pol. A 123, 847 (2013).

[20] M. Turek, Acta Phys. Pol. A 120, 188 (2011).

[21] G.D. Alton, Y. Liu, H. Zaim, S.N. Murray, Nucl. Instrum. Methods Phys. Res. B 211, 425 (2003).

[22] P.A. Hausladen, D.C. Weisser, N.R. Lobanov, L.K. Fifield, H.J. Wallace, Nucl. Instrum. Methods Phys. Res. B 190, 402 (2002).

[23] F.J. Sacherer, IEEE Trans. Nucl. Sci. 18, 1105 (1971).

[24] R. Keller, J.D. Sherman, P. Allison, IEEE Trans. Nucl. Sci. 32, 2579 (1985).

[25] M. Turek, J. Sielanko, P. Franzen, E. Speth, AIP Conf. Proc. 812, 153 (2006).

[26] A. Pyszniak, A. Droździel, M. Turek, A. Latuszyński, D. Maczka, J. Sielanko, Yu.A. Vaganov, Yu.V. Yushkevich, Instrum. Exp. Techn. 50, 552 (2007).

[27] M. Turek, K. Pyszniak, A. Drozdziel, J. Sielanko, A. Latuszynski, D. Maczka, Yu.A. Vaganov, Yu.V. Yushkevich, Instrum. Exp. Techn. 52, 90 (2009).

[28] M. Turek, J. Sielanko, Vacuum 83, S256 (2009).

[29] M. Turek, A. Drozdziel, K. Pyszniak, S. Prucnal, J. Żuk, Przeglad Elektrotechniczny 86, 193 (2010) (in Polish). 